\title{
Acceleration of Slender Bodies of Revolution through Sonic Velocity*
}

\author{
J. D. Colet \\ California Institute of Technology, Pasadena, California \\ (Received January 11, 1954)
}

\begin{abstract}
The linearized theory of slender bodies in arbitrary motion at zero angle of attack has been worked out. The results have been applied to a smooth body accelerating uniformly through sonic velocity. The results theory can be used to estimate the nonlinear or transonic effects.

For an accelerating body, the parameter $\left(b l / c^{2}\right)^{3}$ is important where $2 b=$ acceleration, $2 l=$ length of body, $c=$ sound speed at infinity. For sufficiently high $\left(b l / c^{2}\right)^{t}$, transonic effects can be neglected. Using linearized theory to estimate the ratio of nonlinear terms in the differential equation gives

$$
\lambda=\frac{\text { nonlinear terms }}{\text { significant linear terms }}=\frac{3}{4}(\gamma+1) \frac{\delta^{2}}{\left(b l / c^{2}\right)^{1}}\left\{\log \frac{2}{\delta^{2}}\left(\frac{c^{2}}{b l}\right)^{3}-\frac{9}{4}\right\},
$$

where $\delta=$ thickness ratio of body. The result above is evaluated at the maximum thickness of a symmetric parabolic arc body at the instant it passes through sonic velocity. For $\lambda<1$ transonic effects can be neglected while for $\lambda>1$ they begin to dominate. For practical applications the result shows that there is a possibility of a sufficiently long and slender missile accelerating fast enough to avoid transonic effects (e.g., 50 feet long, 5 percent thick, $3 g$ acceleration). For conventional aircraft, transonic effects will dominate. An interesting side result is that when the acceleration is sufficiently large so that transonic effects do not matter the drag coefficient near sonic speed is independent of the acceleration $\left(C_{D} \doteq 3 \delta^{2}\right.$ for parabolic arc body).
\end{abstract}

\section{INTRODUCTION}

$T$ HE problem discussed here is a special case of the general problem of estimating the validity of linearized theory in the transonic range. The special case studied in detail is that of a slender body of revolution which accelerates uniformly through sonic speed. The method of attacking the problem is applicable to other similar problems of steady and unsteady flow. As a side result the general unsteady slender body expansion is presented.

The work presented here is concerned with the "breakdown" of linearized theory and the necessity for using a more complete theory. Linearized theory is identical in physical and mathematical content with the classical theory of acoustics. ${ }^{1}$ The theory is derived from physical principles plus the assumption that all disturbances are so small that the squares can be neglected. The reason for the breakdown of linearized theory for bodies with thickness can be illustrated as follows. According to linearized theory, all disturbances in the gas travel with a constant speed $c$, the speed of sound. A body flying at an arbitrary speed is continually sending out such disturbances, which, in general, are left behind or escape to the front of the body. For the special case of a body flying steadily at the sonic speed $c$, these disturbances remain with the body, and build up indefinitely. For example, according to linearized theory the pressure on a slender body which starts impulsively

* This paper was presented at a meeting of the American Physical Society in Albuquerque, New Mexico, September 3, 1953. The research was carried out under ARDC Contract AF$18(600)-383$

f Assistant Professor of Aeronautics, California Institute of Technology, Pasadena, California.

${ }^{1}$ Lord Rayleigh, Theory of Sound (Dover Publications, New York, 1945). with sonic speed at the time $t=0$, increases as $\log t$. These results show how linearized theory predicts infinite pressure everywhere on a body flying steadily at sonic speed.

The more accurate theory includes some of the quadratic terms and allows for the fact that the speed of disturbances varies according to local conditions. This refinement prevents the accumulation of disturbances at the body and predicts finite pressures in steady flight at sonic speed. At the same time shock waves must be allowed for in the more exact theory as there is a possibility of small disturbances overtaking one another. The above features are included in the usual transonic theory. Of course, linearized theory does not break down in steady flight only at sonic speed, but there is some neighborhood of Mach numbers about 1 for which it is a poor approximation.

Now, if a body accelerates through sonic speed there is no infinite pressure according to linearized theory, because disturbances do not remain with the body. Nevertheless, there is still some region of acceleration about zero acceleration for which the nonlinear effects are important. Linearized theory becomes valid only for sufficiently high accelerations.

In order to estimate the regions where nonlinear effects become important, the following procedure will be adopted. The equation of motion will be derived under the assumption of small disturbances, but the squared terms will be retained. The relationship of this to the usual acoustic and transonic equations will be shown. The linearized equation will be used to calculate the flow and the results of linearized theory used to estimate the significance of the nonlinear terms. In this way a test of the self-consistency of linearized theory can be obtained. 


\section{SECOND-ORDER EQUATIONS OF MOTION}

Consider a system of coordinates $(x, y, z, t)$ in which air is at rest at infinity. It will be assumed that any local, normal Mach numbers measured relative to any shock waves that occur, are not very different from 1 . Thus entropy changes $\sim\left(M_{x}{ }^{2}-1\right)^{3}$ are neglected. It follows from Crocco's vortex theorem ${ }^{2}$ that the flow can be regarded as irrotational. These assumptions mean that the dependent variables can be reduced to $\mathbf{q}=\mathbf{v e -}$ locity, $P=$ pressure, and $\rho=$ density, where $P, \rho$ are always related by the isentropic law

$$
P / P_{0}=\left(\rho / \rho_{0}\right)^{\gamma},
$$

where subscript 0 denotes conditions at infinity, and $\gamma=$ ratio of specific heats. Furthermore, a potential $\phi$ exists so that

$$
\mathbf{q}=\operatorname{grad} \phi .
$$

Now, assuming small disturbances, let

$$
P=P_{0}(1+p), \quad \rho=\rho_{0}(1+s), \quad p, s \ll 1 .
$$

Then (1) becomes, to the second order (that is, neglecting cubed terms),

$$
p=\gamma s+\frac{\gamma(\gamma-1)}{2} s^{2}
$$

The equation for conservation of momentum, in view of (2)

$$
\mathbf{q}_{t}+\nabla\left(\frac{q^{2}}{2}\right)=-\frac{1}{\rho}-\nabla p,
$$

can be integrated by virtue of (2) and (4) to give a second-order Bernoulli law,

$$
s=-\frac{1}{c^{2}} \phi_{t}-\frac{1}{2 c^{2}}\left\{(\nabla \phi)^{2}+(\gamma-2) \frac{\phi_{t}{ }^{2}}{c^{2}}\right\},
$$

where $c^{2}=\gamma\left(P_{0} / \rho_{0}\right)=$ speed of sound at infinity. Thus, by using Eqs. (3), and (6), the kinematic requirements of continuity give the equation of motion for the velocity potential

$$
\rho_{i}+\operatorname{div} \rho \mathbf{q}=0
$$

and can be written:

$$
\frac{1}{c^{2}}\left\{1+(\gamma-1) \frac{\phi_{t}}{c^{2}}\right\} \phi_{t t}+\frac{1}{c^{2}} \frac{\partial}{\partial t}(\nabla \phi)^{2}-\Delta \phi=0 .
$$

Equation (8) is the fundamental equation of motion, including all squared terms in $\phi$ but neglects all cubed or higher terms. The form of (8) is chosen for convenience; multiplying through by $1+$ (small quantity) gives equivalent forms. As will be shown in the next section, terms like $\phi_{t} \phi_{t t}$ and $\phi_{x} \phi_{x t}$, which account for the steepening of wave fronts in this system of coordinates,

${ }^{2}$ R. Sauer, Theoretical Gas-Dynamics (Edwards Brothers, Inc., Ann Arbor, Michigan, 1947). account for the change of type of the equations or the fact that stream tubes have throats at sonic speed, when viewed in a steadily moving coordinate system.

\section{RELATIONSHIP TO ACOUSTICS AND TRANSONIC THEORIES}

If all squared terms are omitted in (8), (6), and (4), the classical equations of acoustics are obtained,

$$
\begin{gathered}
\left(1 / c^{2}\right) \phi_{t t}-\Delta \phi=0, \\
p / \gamma=s=-\left(1 / c^{2}\right) \phi_{l} .
\end{gathered}
$$

The speed of all disturbances is constant and equal to $c$.

Next, consider Eq. (8) in a system of coordinates moving at a constant speed $U$ in the negative $x$ direction. For steady flow in the new coordinate system, $\partial / \partial t$ in Eq. (8) is replaced by $U(\partial / \partial X)$, so that

$$
\begin{array}{r}
\left(M^{2}-1\right) \phi_{X X}-\left(\phi_{y y}+\phi_{z z}\right)+\frac{(\gamma-1) M^{2}+2}{c^{2}} U \phi_{X} \phi_{X X} \\
+\frac{U d \partial}{c^{2} \partial X}\left(\phi_{y}{ }^{2}+\phi_{z}{ }^{2}\right)=0,
\end{array}
$$

where

$$
X=x+U t, \quad T=t,
$$

$M=U / c=$ free stream Mach number in $(X, t)$ system.

When squared terms are neglected, (11) is the usual linearized equation of steady subsonic or supersonic flow. But when the nonlinear terms containing $\phi_{X} \phi_{X X}$ are retained in (11), on the grounds that the results of linearized theory hint that they are much larger than the others, the usual equation of transonic flow is obtained (for $M \doteq 1$, the factor $\left(1 / c^{2}\right)\left\{(\gamma-1) M^{2}+2\right\}$ can be replaced by $\left(\gamma+1 / a^{*}\right) M^{2}$, as in reference 3$)$.

\section{ESTIMATION OF NONLINEAR EFFECTS}

In order to show how to estimate the relative importance of nonlinear effects, consider the case of steady flow as covered by Eq. (11). The reason for the breakdown of linearized theory at sonic speed is the vanishing of the term $\left(M^{2}-1\right) \phi_{X X}$ in the equation. The size of nonlinear effects can be measured by the ratio of the main nonlinear term in Eq. (11) to this term which vanishes at $M=1$, namely,

$$
\begin{array}{r}
\lambda=\frac{\text { nonlinear terms }}{\text { linear terms }}=\frac{\left[(\gamma-1) M^{2}+2 / c^{2}\right] U \phi_{X} \phi_{X X}}{\left(1-M^{2}\right) \phi_{X X}} \\
\doteq \frac{(\gamma+1) M^{2} \phi_{X}}{1-M^{2}} \frac{1}{U} .
\end{array}
$$

Note that $\lambda=1$ corresponds to an estimate of the critical Mach number, that is, $\lambda=1$ means the local Mach number is one.

3 A. Busemann, "Application of Transonic Similarity," National Advisory Committee for Aeronautics TN 2687 (1952). 
Now, linearized theory itself can be used to evaluate $\lambda$ in (12). For example, in the case of two-dimensional flow past nonlifting air-foil of thickness ratio $\delta$, the pressure coefficient

$$
c_{p} \doteq-2 \frac{\phi_{X}}{U} \sim \frac{\delta}{\left(1-M^{2}\right)^{\frac{1}{3}}} M<1,
$$

the usual Prandtl-Glauert rule. ${ }^{4}$ Using the result of (13) in (12) shows that the transonic similarity parameter $\xi^{3}$ should be small compared to 1 for linearized theory to be valid, or

$$
\lambda-\frac{(\gamma+1) M^{2} \delta}{\left(1-M^{2}\right)^{3}}=\xi^{\xi}<1 .
$$

When $\lambda$ gets close to 1 the nonlinear terms are becoming important, and when $\lambda>1$ they definitely dominate. It is slightly more complicated to carry out the estimate for the case of a body of revolution as the simple proportionality of (13) no longer holds. However, the result can be illustrated by applications of slender-body theory ${ }^{4}$ to the special case of a symmetric parabolic arc body of revolution of thickness ratio $\delta$. The result is that

$$
\lambda=(\gamma+1) M^{2} \frac{\delta^{2}}{1-M^{2}}\left\{2 \log \frac{2}{\delta\left(1-M^{2}\right)^{1}}-3\right\}
$$

when evaluated at the maximum thickness point. The result is typical for smooth bodies of revolution, but the constants vary a little with shape. Equation (15) shows how, as would be expected, a body of revolution can fly much closer to sonic speed than its two-dimensional counterpart without experiencing transonic effects. Figure 1 gives a plot of the results of $\mathrm{Eq}$. (15) for several thickness ratios.

Now the same reasoning can be carried over to the general unsteady case in the transonic range. The transonic effects can be measured by the ratio of nonlinear to significant linear terms. The significant linear terms are $\left(1 / c^{2}\right) \phi_{t t}-\phi_{x x}$, since under translation of axes these terms become $\left(M^{2}-1\right) \phi_{X X X}$. Thus, from Eq. (8),

$$
\lambda=\frac{\left(\gamma-1 / c^{4}\right) \phi_{t} \phi_{t t}+\left(2 / c^{2}\right) \phi_{x} \phi_{x t}}{\left(1 / c^{2}\right) \phi_{t t}-\phi_{x x}} .
$$

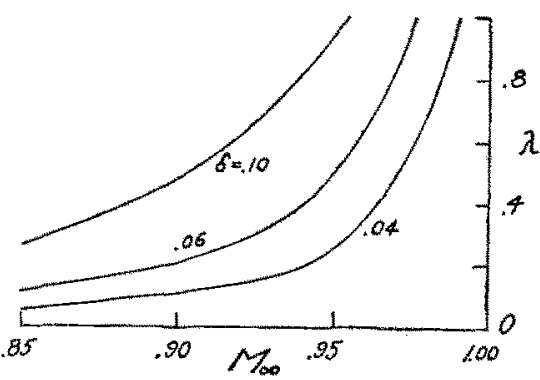

Fic. 1. Transonic parameter $\lambda$ is Mach number $M_{\infty}$ at maximum thickness symmetric parabolic body.

4W. Sears and M. Adams, J. Aeronaut. Sci. 20, 85 (1953).

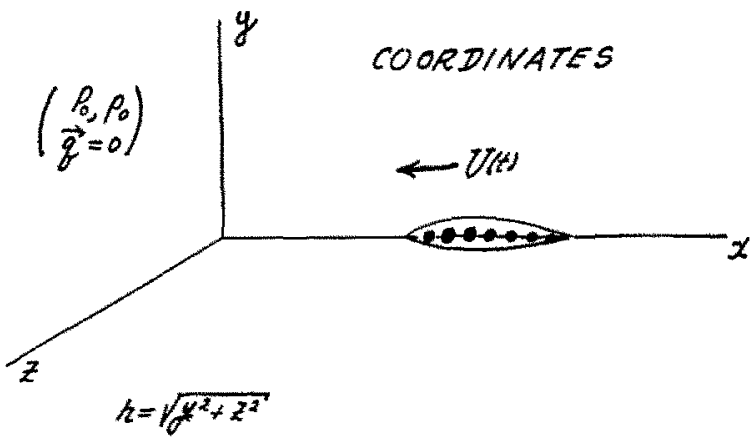

Frg. 2. Coordinate system.

\section{UNSTEADY SLENDER-BODY THEORY}

As before, the values of $\lambda$ given by (16) are to be computed on the basis of linearized theory. For a body of revolution at zero angle of attack, the potential is given by the solutions of

$$
\frac{1}{c^{2}} \phi_{t t}-\phi_{x x}=\phi_{r r}+\frac{1}{r} \phi_{r}
$$

where $r=\left(y^{2}+z^{2}\right)^{2}$. A body of revolution moving along its axis (Fig. 2) can be represented by a distribution of sources, which vary in time, along the axis of flight. The potential is represented by an integral (expressing the superposition of spherical waves emanating from each point on the axis) of the retarded values of the source strength $S(x, t)$ :

$$
-4 \pi \phi(x, r, t)=\int_{-\infty}^{\infty} \frac{s\left\{\xi, t-\frac{\left[(x-\xi)^{2}+r^{2}\right]^{1}}{c}\right\}}{\left[(x-\xi)^{2}+r^{2}\right]^{t}} d \xi,
$$

The source strength is zero outside the body so that the integral in (18) covers only those values of $\xi$ which are common to the flight path and the surface of the retrograde Mach cone, $\tau=t-(1 / c)\left[(x-\xi)^{2}+r^{2}\right]^{1}$, as shown in Fig. 3. There are integrals for the recent past $x_{1}$ to $x_{2}$, and the distant past $x_{3}$ to $x_{4}$, etc.

The source integrals can be approximated near the axis by using the fact that when $r$ is small, the main contribution comes from the neighborhood of $x=\xi$. The integral for the recent past can be analyzed for small $r$ by splitting it into

$$
\int_{x 1}^{x-\varepsilon(r)}, \int_{x-\varepsilon(r)}^{x+\varepsilon(r)}, \int_{x+\varepsilon(r)}^{x_{2}} .
$$

The expansion for the middle integral can be obtained by introducing the variable $\sigma$ as $x=\xi+r \operatorname{sh} \sigma$, and then expanding in powers of $r$. A further expansion in powers of $r / \epsilon$ is possible since we assume $\epsilon>r$. For the outside integrals, expansions of $S$, and the square root in powers of $r$ can be made. Integration by parts in the outside integrals produces a result, the dominant terms 
of which are independent of $\epsilon$, as $r$ and $\epsilon$ approach zero. The final result is the asymptotic expansion of the source distribution near the axis:

$$
\begin{gathered}
\phi(x, r, t)=\frac{1}{2 \pi} S(x, t) \log _{2}-\frac{1}{4 \pi}\left\{S\left(x_{1}, t-\frac{x-x_{1}}{c}\right)\right. \\
\left.\times \log \left(x-x_{1}\right)+S\left(x_{2}, t-\frac{x_{2}-x}{c}\right) \log \left(x_{2}-x\right)\right\} \\
-\frac{1}{4 \pi} \int_{x_{1}}^{x_{2}}\left\{\frac{1}{c} S_{t}\left(\xi, t-\frac{|x-\xi|}{c}\right)\right. \\
\left.+\operatorname{sgn}(x-\xi) S_{x}\left(\xi, t-\frac{|x-\xi|}{c}\right)\right\} \log |x-\xi| d \xi \\
-\frac{1}{4 \pi} \int_{x_{3}}^{x_{4}} \frac{S\left(\xi, t-\frac{|x-\xi|}{c}\right)}{|x-\xi|} d \xi, \text { etc. }+\cdots+O\left(r^{2} \log r\right),
\end{gathered}
$$

where $x_{1}(x, t), x_{2}(x, t)$ are the values of $\xi$ in the recent past at which $\tau=t \mp(x-\xi) / c$, respectively, intersect the boundaries of the flight path (Fig. 3), and $x_{3}, x_{4}$ are similar values in the distant past.

Equation (19) is the general slender-body expansion. In most cases only the integrals in the recent past $\left(x_{1}, x_{2}\right)$ need be considered. The physical interpretation of Eq. (19) is as follows: the dominant $\log r$ term is the potential of a source in two-dimensional incompressible flow. This means that locally the conservation of mass can be computed in a cross-section plane. The next terms in the expansion are independent of $r$ and represent the effect of the sources at a distance from the local neighborhood. All the higher terms in the expansion can be obtained by the substitution of the first terms of (19) in the left-hand side of Eq. (17). This procedure results in terms like $r^{2} \log r, r^{2}$ etc. It can be expected that the first terms of the asymptotic expansion, that is Eq. (19), are a good approximation near a smooth slender body except in the neighborhood of the ends.

Using the slender-body approximation of (19) the source strength $S(x, l)$ is easily determined by the requirement that the flow be tangent at the surface. Within linearized theory this boundary condition is

where

$$
\phi_{r}(x, R, t)=U(t)(\partial R / \partial x)(x, t)
$$

$r=R(x, l)$ is the equation of the body shape,

$U(t)=$ velocity of the body in the negative $x$ direction.

For a body of fixed shape we obtain, from (20) and (19),

$$
S(x, l)=U(l) A^{\prime}(X)
$$

where

$X=x+\int_{0}^{t} U d \tau$ is a coordinate fixed in the body. $A(x)=$ cross-section area distribution.

The fundamental result (21), due to Frankl, ${ }^{5}$ states that the source strength for arbitrary motion is the same as that for incompressible flow and is equal to the instantaneous velocity times the rate of change of crosssection area along the body. Once the source strength is known the velocities and pressure can be computed of course.

\section{APPLICATION TO A BODY UNIFORMLY ACCELERATING THROUGH SONIC SPEED}

Consider a body which passes through sonic speed at $t=0$ and lies between $-l$ and $+l$. Let the acceleration be $2 b$ so that the velocity is

$$
U(t)=c+2 b t .
$$

The limits of integration $x_{1}, x_{2}$ are then given by

$$
\left\{\begin{array}{r}
x-x_{1}=c t+\frac{c^{2}}{b}\left\{1-\left[1-\frac{b}{c^{2}}(x+l-c t)\right]^{\frac{1}{3}}\right\} \\
=\frac{x+l+c t}{2}+O(b) \\
x_{2}-x=c t+c\left(\frac{l-x-c t}{b}\right)^{\frac{1}{2}}
\end{array}\right\}
$$

Furthermore, assume the body is pointed (or has zero slope) at the ends, so that

$$
S\left(x_{1}, t-\frac{x-x_{1}}{c}\right)=S\left(x_{2}, t-\frac{x_{2}-x}{c}\right)=0 .
$$

Then, using (23) and (21), the velocity potential can be written out from (19). If now we consider cases in which the acceleration parameter $b l / c^{2} \ll 1$, which is

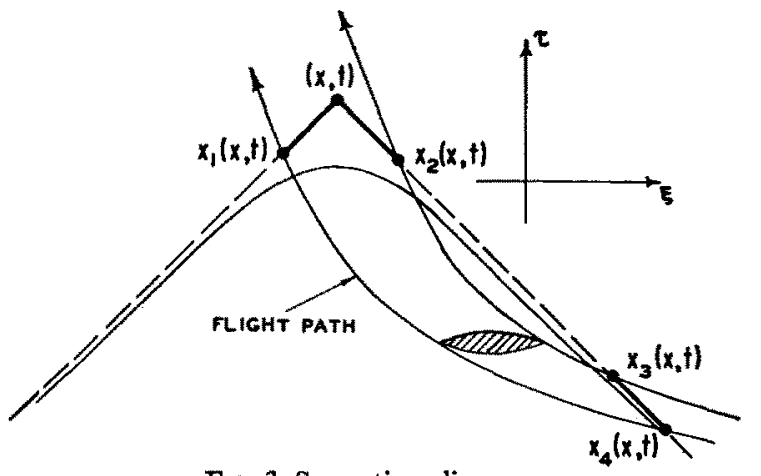

Fro. 3. Space-time diagram.

5 F. I. Frank], Effect of the Acceleration of Elongated Bodies of Revolution upon the Resistance in Compressible Flow, National Advisory Committee for Aeronautics TM 1230 (1949); translation of the Russian paper in Prikl. Mat. i. Mekh 10 (1946). 
true in all practical examples, a further expansion of Eq. (19) can be made. The final result is

$$
\begin{aligned}
& -4 \pi \phi(x, r, t)=c A^{\prime}(x+c t) \underset{r^{2}}{\log \frac{2 c}{b}}\left(\frac{l-x-c t}{b}\right)^{\frac{1}{2}}(l+x+c t) \\
& +\sum_{n=1}^{\infty} \frac{1}{n \Gamma(n+1)}\left\{\frac{1}{2}(l-x-c t)^{n}+(-)^{n}(l+x+c t)^{n}\right\} \\
& \times\left(\frac{\partial}{\partial x}\right)^{n} A^{\prime}(x+c t)-c t\left[b(l-x-c t)^{\frac{1}{b}}\right. \\
& \times \sum_{n=0}^{\infty} \frac{(l-x-c t)^{n}}{\left(n+\frac{1}{2}\right) \Gamma(n+1)}\left(\frac{\partial}{\partial x}\right)^{n} A^{\prime \prime}(x+c t) \\
& \quad+f n(x+c t ; \sqrt{ } b)+o(b \log b) .
\end{aligned}
$$

From (10), the main term in the pressure distribution contains $\phi_{t}$. On the surface of the body, at the instant of passing through sonic $t=0$, (24) gives

$$
\begin{array}{r}
-\frac{4 \pi}{c^{2}} \phi_{t}(x, R(x), 0)=A^{\prime \prime}(x) \log \frac{2 \pi c}{A(x)}\left(\frac{l-x}{b}\right)^{\frac{1}{2}}(l+x) \\
+\sum_{n=1}^{\infty} \frac{1}{n \Gamma(n+1)}\left\{\frac{1}{2}(l-x)^{n}+(-)^{n}(l+x)^{n}\right\} \\
\times\left(\frac{\partial}{\partial x}\right)^{n} A^{\prime \prime}(x)+O(\sqrt{ } b) .
\end{array}
$$

From (25) it can be seen that, for any body, as the acceleration $b \rightarrow 0$ the pressure becomes logarithmically infinite, indicative of the manner in which the linearized theory breaks down. It can also be seen that the drag coefficient at sonic, calculated from the linearized pressure formula

$$
c_{p}=-\frac{2}{U^{2}} \phi_{t}-\frac{1}{U^{2}} \phi_{r}{ }^{2}+\cdots
$$

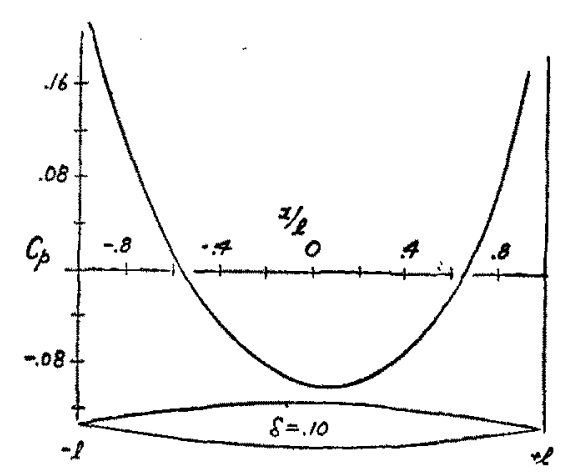

FIg. 4. Pressure distribution at sonic speed; uniform acceleration, $b l / c^{2}=0.026$. is independent of the acceleration $b$. (Its value is not the same as that of steady supersonic slender-body theory as $M \rightarrow 1+$.) This result can be interpreted as follows. For slow accelerations, nonlinear effects are important, and there is a definite drag depending on the acceleration. As the acceleration increases, the drag coefficient at sonic decreases. For sufficiently large accelerations, linearized theory is valid and the drag coefficient approaches its asymptotic value independent of the acceleration.

As an illustration consider a symmetric parabolic arc body of thickness ratio $\delta$, whose shape is

$$
r=R(x)=\delta l\left(1-\frac{x^{2}}{l^{2}}\right)
$$

Then the pressure coefficient at sonic can be calculated from (26) and (25) to give

$$
\begin{array}{r}
\frac{c_{p}}{2}=\delta^{2}\left(\frac{3 x^{2}}{l^{2}}-1\right) \log \left\{\frac{2}{\delta^{2}}\left(\frac{c^{2}}{b l}\right)^{\frac{3}{2}} \frac{1}{\left.\left(1+\frac{x}{l}\right)\left(1-\frac{x}{l}\right)^{\frac{3}{2}}\right\}}\right. \\
+\frac{\delta^{2}}{4}\left\{9-6-\frac{x}{l}-35 \frac{x^{2}}{l^{2}}\right\}
\end{array}
$$

A special case of this formula is plotted in Fig. 4. The slight unsymmetry of the pressure distribution results in a drag

$$
C_{D}{ }^{*}=2.9 \delta^{2}\{1+O(\sqrt{ } b)\}
$$

Finally the range of validity of linearized theory can be estimated by using (24) to evaluate the ratio $\lambda$,

$$
\begin{aligned}
\lambda \doteq \frac{(\gamma+1)\left(\phi_{t} \phi_{t t} / c^{4}\right)}{\left(1 / c^{2}\right) \phi_{t t}-\phi_{x x}}=\frac{3}{4}(\gamma+1) & \frac{\delta^{2}}{\left(b l / c^{2}\right)^{\frac{1}{2}}} \\
& \left\{\log _{\delta^{2}}^{2}\left(\frac{c^{2}}{b l}\right)^{\frac{1}{2}}-\frac{9}{4}\right\}
\end{aligned}
$$

It can be seen from (30) that for a fixed $\delta$, the accelera-

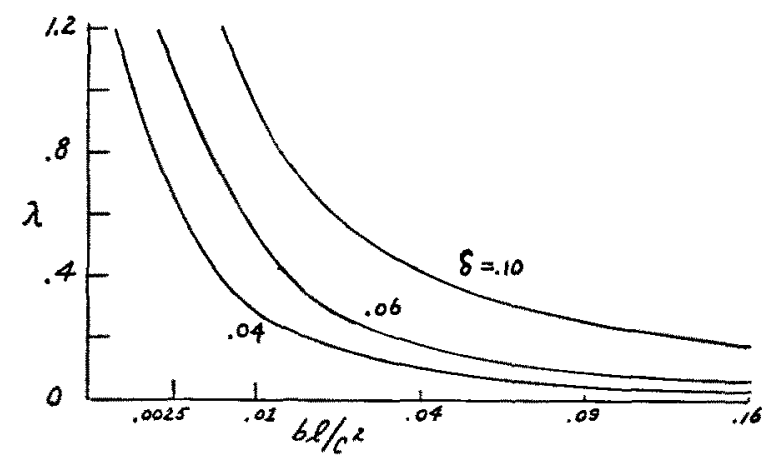

FIG. 5. Transonic effect parameter $\lambda v s$ acceleration parameter $\mathrm{bl} / \mathrm{c}^{2}$ at maximum thickness of symmetric parabolic arc body $M=1$. 
tion parameter $b l / c^{2}$ can always be chosen large enough so that $\lambda<1$, and linearized theory is valid. A plot of Eq. (30) is given in Fig. 5. The numerical results show that for practical applications, it is possible for a sufficiently long and slender missile (for example, $50 \mathrm{ft}$ long, $\delta=0.05,3 g$ acceleration) to accelerate through sonic without experiencing transonic effects, while it is not possible for conventional aircraft to do so.

As a concluding remark, the author wishes to thank Dr. George Solomon for his able assistance.

\title{
Use of Space Charge in Electron Optics
}

\author{
E. A. AsH \\ Nuclear Parlicle Laboratory, Queen Mary College, London, England
}

(Received December 28, 1953)

\begin{abstract}
The electron optical properties of a cylindrical space charge cloud are derived. The possibility of achieving a system free from either spherical or chromatic aberration by combining a space charge lens with a space charge free converging lens is examined. It is concluded that the achromatization of a thin electric or magnetic lens is possible only if the resultant action of the combination is divergent. The correction of the spherical aberration of a high quality lens, such as an electron microscope objective, is shown to be impossible on account of electron interaction effects.
\end{abstract}

\section{INTRODUCTION}

$T$ HE design of space charge free electron optical lenses with prescribed properties is restricted by the fact that the lens fields must be solutions of Laplace's equation. This limitation does not preclude the construction of a two-dimensional lens ${ }^{1.2}$ (imaging coplanar line elements) free from spherical aberration, but in the case of a three-dimensional lens (imaging elements of area) it is not possible, as has been shown by $\mathrm{O}$. Scherzer, ${ }^{3}$ to eliminate either the spherical aberration (s.a.) or the chromatic aberration (c.a.) completely. The level to which the s.a. (which is always positive on the usual convention) can be reduced is limited by the maximum strength of the lens (magnetic or electric) which can be achieved in practice.

D. Gabor ${ }^{4}$ has shown that if the fields are not required to obey Laplace's equation, it is possible to find a solution with the properties of an "ideal lens," which would form an undistorted image (though with unit magnification) of any finite volume element in the object space. The realization of such a lens would require an infinite spherically symmetric charge distribution, involving charges of both signs. Gabor ${ }^{5}$ showed further the existence of a lens which has "semi-ideal" properties in the sense that it can image an element of area without s.a. and with a magnification greater than unity. Such a lens could be realized by an axially symmetric charge distribution of negative charges only.

Since an isolated electron cloud acts as a divergent lens, it would seem by analogy with optics that it might

\footnotetext{
${ }^{1}$ W. Glaser, Nature 162, 455 (1948).

${ }^{2}$ N. Wax, J. Appl. Phys. 24, 727 (1953)

${ }^{3}$ O. Scherzer, Z. Physik 101, 593 (1936).

D. Gabor, The Electron Microscope (Hulton Press, London, 1944).

D. Gabor (unpublished).
}

also be possible to achieve an achromatic system by combination with a converging lens. The elimination of s.a. and of c.a. by the use of space charge has been briefly considered by a number of authors, who have been well aware of the magnitude of the technical problems involved.

In the course of an experimental investigation on electron interaction effects, ${ }^{6}$ it was found possible to produce a dense, stable electron cloud by very simple means. The essential features of the system, which are shown in Fig. 1, consisted of a hollow cylindrical cathode surrounding a concentric grid, held at a positive potential. It was shown, both theoretically and experimentally, that by the introduction of a weak axial magnetic field the radial distribution of space charge density could be adjusted so as to give a strong negative spherical aberration to the divergent lens formed by the space charge. It appeared that the technical difficulties which would be involved in perfecting this device to a degree where it could be of use in precision electron optics were not insuperable. In the light of these experiments the possibility of the use of space charge as correcting elements was re-examined.

When first proposing the correction of s.a. by means of space charge, Gabor observed that it was dependent on the electron interaction effect being sufficiently small to allow the representation of the electron cloud by a smooth space charge distribution. The possibility of the correction of c.a. depends on the variation, with the velocity of the electron beam, of the focal length of the space charge lens. The present investigation has led to the negation of both these possibilities. It is the purpose of this paper to show that the use of space charge as

E. A. Ash and D. Gabor Proc. Roy. Soc. (London) A (to be published). 